\title{
Chinese Expert Consensus on the Diagnosis and Treatment of Castration-Resistant Prostate Cancer (2019 Update)
}

This article was published in the following Dove Press journal: Cancer Management and Research

\author{
Yao Zhu ${ }^{1,2}$ \\ Dingwei $\mathrm{Ye}^{1,2}$ \\ On behalf of the Expert \\ Group \\ 'Department of Urology, Fudan \\ University Shanghai Cancer Center, \\ Shanghai, People's Republic of China; \\ ${ }^{2}$ Department of Oncology, Shanghai \\ Medical School, Fudan University, \\ Shanghai, People's Republic of China
}

\begin{abstract}
Currently, the basic treatment of advanced prostate cancer is still endocrine therapy, but almost all patients eventually progress to castration-resistant prostate cancer (CRPC). In 2016, the Chinese Expert Consensus on the Diagnosis and Treatment of CRPC which aimed to help Chinese clinicians formulate treatment plans for CRPC was published. In this 2019 update, the 2016 version was updated with the aim of providing a more appropriate reference for clinical practice, standardizing CRPC patient management, and facilitating decision-making. The consensus is evidence-based and reviews the optimal therapeutic recommendations for CRPC management in China by taking into consideration the clinical characteristics of Chinese patients; drug availability, efficacy and safety; and recent advancements and developments in the international medical arena.
\end{abstract}

Keywords: prostate cancer, castration-resistant, metastases, treatment, expert consensus

\section{Introduction}

The number of prostate cancer patients in China is gradually increasing, with most cases presenting with metastasis at the time of initial diagnosis. At present, the basic treatment for advanced prostate cancer is still endocrine therapy, but almost all patients eventually progress to castration-resistant prostate cancer (CRPC). ${ }^{1}$ The median survival time of CRPC patients with metastasis is less than 2 years. In 2016, the Chinese Expert Consensus on the Diagnosis and Treatment of CastrationResistant Prostate Cancer was officially published, helping and guiding Chinese clinicians in designing a more reasonable approach to the treatment plan for CRPC. Thereafter, the treatment patterns for CRPC experienced some changes internationally, and some new drugs were approved for this indication. Therefore, clinicians urgently required a more updated set of guidelines which would not only incorporate recent advances in management concepts on an international level, but also accommodate and conform to the actual situation of our country, and hence effectively attend to the needs of clinical practice. Consequently, the Expert Group updated the 2016 version of the consensus, with the aim of providing a more appropriate reference for clinical practice, standardizing CRPC patient management, and facilitating decision-making, and ultimately improving the quality-of-life and survival of patients.

The levels of evidence in this consensus are divided into three: A, B and C (Table 1). Revisions were also made to the GRADE classification system. ${ }^{2}$ Members of the Expert
Correspondence: Dingwei Ye Department of Oncology, Shanghai Medical School, Fudan University, No. 270 Dongan Road, Shanghai 200032, People's Republic of China

Tel +861370166357I

Email dwyeli@I63.com 
Table I Levels of Evidence and Grades of Recommendation

\begin{tabular}{|l|l|}
\hline $\begin{array}{l}\text { Level of } \\
\text { Evidence }\end{array}$ & Description \\
\hline $\begin{array}{l}\text { A. High } \\
\text { quality }\end{array}$ & $\begin{array}{l}\text { Further research is unlikely to change the credibility } \\
\text { of the outcome of the evaluation }\end{array}$ \\
\hline $\begin{array}{l}\text { B. Moderate } \\
\text { quality }\end{array}$ & $\begin{array}{l}\text { Further research may have a significant impact on } \\
\text { the reliability of the outcome of the efficacy } \\
\text { evaluation }\end{array}$ \\
\hline C. Low quality & $\begin{array}{l}\text { Further research is likely to affect the credibility of } \\
\text { the outcome of the efficacy assessment, and is likely } \\
\text { to change the outcome of the assessment. }\end{array}$ \\
\hline
\end{tabular}

Group were selected and represent experts in the urological field in China. Each expert selected items that required updating from the 2016 version and provided the latest evidence and research. Then the Expert Group held workshops to discuss the primary review results for each update item. The updates that were then reviewed and further discussed at a consensus conference attended by all experts. Based on these discussions, the Expert Group wrote the 2019 consensus.

\section{Definition and Epidemiology of CRPC in China \\ Definition}

CRPC is defined as disease progression in the setting of castrate levels of testosterone. Specifically, it is defined as castration serum testosterone levels $(<50 \mathrm{ng} / \mathrm{dL}$ or $<1.7$ $\mathrm{nmol} / \mathrm{L}$ ) and at least one of the following conditions: ${ }^{3}$ (1) biochemical progression [three consecutive rises in prostate-specific antigen (PSA) 1 week apart resulting in two $50 \%$ increases over the nadir and a PSA $>2 \mathrm{ng} / \mathrm{mL}$; and (2) radiological progression [appearance of two or more new bone lesions on a bone scan or a soft tissue lesion using the Response Evaluation Criteria in Solid Tumors (RECIST)]. Symptomatic progression alone cannot be diagnosed as CRPC and requires further evaluation.

\section{Epidemiological Statistics}

Prostate cancer is the second most common cancer (accounting for $13.5 \%$ of all cancers) and the fifth leading cause of cancer deaths in men worldwide (accounting for $6.7 \%$ of all cancer deaths). ${ }^{4}$ In 2018 , it was estimated that there were nearly 1.3 million new cases of prostate cancer worldwide, and 359,000 prostate cancer-related deaths. ${ }^{4}$ The incidence of prostate cancer in China has been increasing over the recent years and in 2015, an estimated
60,300 new cases and 26,600 deaths from prostate cancer occurred in China. ${ }^{5}$

Most prostate cancer patients in China present with metastasis at the time of initial diagnosis. At present, endocrine therapy is still the mainstay of treatment for patients with advanced prostate cancer, but after 18 to 24 months of endocrine therapy, almost all patients progress to $\mathrm{CRPC}^{1}$

\section{Current Treatments for CRPC}

Based on their different mechanisms of action, drugs for CRPC treatment include novel endocrine therapies, chemotherapy agents, immunotherapy agents, targeted therapies, and bone metastasis therapies, some of which have already been introduced into clinical practice in China (Table 2). Table 2, which shows the main therapeutic drugs used for CRPC in China, was compiled from the registration and information platform for clinical trials of drugs in China. The website is: http://www.chinadrugtrials.org.cn/eap/clini caltrials.searchlist. There has been some experience in China with novel endocrine agents such as abiraterone, cytotoxic drugs such as docetaxel, mitoxantrone, and estramustine, and zoledronic acid for bone metastasis, and a number of newer agents are ready to be launched but there is limited clinical experience with them, such as the new generation of antiandrogen drugs (eg apalutamide and enzalutamide),

Table 2 The Main Therapeutic Drugs Used for CRPC in China

\begin{tabular}{|c|c|c|c|}
\hline Year & Available in China & $\begin{array}{l}\text { Testing } \\
\text { Complete; } \\
\text { Evaluation in } \\
\text { Progress }\end{array}$ & $\begin{array}{l}\text { Applying for } \\
\text { Conducting } \\
\text { Clinical Research } \\
\text { in China }\end{array}$ \\
\hline $\begin{array}{l}\text { Before } \\
2000\end{array}$ & $\begin{array}{l}\text { Estrostatin (1988) } \\
\text { Bicalutamide (1999) }\end{array}$ & & \\
\hline $2000-2005$ & Zoledronic acid* & & \\
\hline $2006-2010$ & $\begin{array}{l}\text { Docetaxel (approved } \\
\text { in 2008) }\end{array}$ & & \\
\hline $2011-2015$ & $\begin{array}{l}\text { Abiraterone acetate } \\
\text { (2015) }\end{array}$ & & \\
\hline $2016-$ & & $\begin{array}{l}\text { Radium-223 } \\
\text { chloride } \\
\text { Denosumab } \\
\text { Orteronel } \\
\text { (TAK-700) }\end{array}$ & $\begin{array}{l}\text { Enzalutamide } \\
\text { Apalutamide } \\
\text { Cabazitaxel } \\
\text { Ipatasertib (AKT } \\
\text { inhibitor) } \\
\text { Atezolizumab (PD- } \\
\text { I inhibitor) } \\
\text { Fluzoparib (PARP } \\
\text { inhibitor) }\end{array}$ \\
\hline
\end{tabular}

Note: *Imported zoledronic acid was withdrawn from the Chinese market in 2018. 
radium-233, and denosumab, which is used in the treatment of bone metastasis. The ODM-201 and ${ }^{177} \mathrm{Lu}$ clinical trials have shown that these drugs are effective and deserve attention in the future.

Nevertheless, there are some drugs available in other countries that are not yet approved or marketed in China; for instance, cytotoxic drugs such as cabazitaxel, the autologous active cellular immunotherapy (sipuleucel-T), and inhibitors of the programmed cell death molecule-1 (PD-1) and its ligand (PD-L1). In addition, the value of targeted therapies such as the poly ADP-ribose polymerase (PARP) inhibitor olaparib is also being explored in CRPC.

This consensus is evidence-based and presents the optimal therapeutic recommendations for CRPC management in China by taking into consideration the clinical characteristics of Chinese patients; drug availability, efficacy and safety; as well as recent advancements and developments in the international arena.

\section{Clinical Management of Non-Metastatic (NM) CRPC Definition and Diagnosis of NM-CRPC}

Non-metastatic castration-resistant prostate cancer (NMCRPC), also known as M0 CRPC, refers to prostate cancer that has exhibited biochemical progression after drug or surgical castration but is without evidence of distant metastasis through conventional radiological examination.

The diagnosis of NM-CRPC requires simultaneous fulfillment of the following 3 conditions: ${ }^{3}$ (1) castration levels of serum testosterone $(<50 \mathrm{ng} / \mathrm{dL}$ or $<1.7 \mathrm{nmol} / \mathrm{L})$; (2) three consecutive rises in PSA 1 week apart resulting in two $50 \%$ increases over the nadir and a PSA $>2 \mathrm{ng} / \mathrm{mL}$; and (3) a bone scan with or without thoracic, abdominal or pelvic CT (or MRI in patients with CT contraindications) that does not show signs of new lesion(s).

\section{Management and Monitoring of NM-CRPC}

About one-third of patients with NM-CRPC will have clinically detectable metastasis 2 years after diagnosis. The baseline PSA level, the rate of rise of PSA, and the PSA double time (PSA-DT) are independent predictive factors for the risk of metastasis, which is related to the time of the first bone metastasis, survival without bone metastasis, and overall survival. ${ }^{6,7}$ Patients receiving androgen deprivation therapy (ADT) should have regular testing of their PSA level and radiological examinations to detect CRPC and early-stage metastatic lesions. For patients with a high risk of metastasis, it is recommended to increase the frequency of PSA testing. Rcommendations for Management and Monitoring of NM-CRPC, see Box 1.

\section{Box I Management and Monitoring of NM-CRPC}

Recommendations
Recommendation I: Prostate cancer patients undergoing
continuous ADT treatment are advised to have follow-up PSA
testing every 3 to 6 months; for those at high risk for disease
progression, PSA testing is recommended every 3 months.
Recommendation 2 : When asymptomatic patients present with
a PSA level $\geq 2 \mathrm{ng} / \mathrm{mL}$, it is advisable to perform a bone scan with or
without a CT scan; if the results are negative, it is recommended to
repeat the PSA test every 3 months. When the PSA level increases
to more than $\geq 5 \mathrm{ng} / \mathrm{mL}$ or the PSA level doubles, a bone scan is
recommended. In patients with a PSA $\geq 5 \mathrm{ng} / \mathrm{mL}$ with negative
findings on CT or bone scans, PSA follow-up is recommended
every 3 months, and when the PSA level doubles, a bone scan is
recommended. In the event of manifestations such as bone pain,
irrespective of the PSA level, bone and CT scans are
recommended. 8,9

\section{Treatment}

Apart from ADT-related symptoms, patients with NMCRPC normally do not present with other manifestations, and maintain a state of relative physical health. ${ }^{10}$ However, in cases of bone metastasis, the survival outcome of patients decreases significantly. Hence, it becomes more meaningful to increase the metastasis-free survival and delay the incidence of metastasis. Thus, there has been much focus on the treatment of NM-CRPC, and there have been tremendous advances over the last few years.

\section{Localized Non-Progressive NM-CRPC \\ Apalutamide}

Apalutamide is the first approved drug for the treatment for NM-CRPC. It is a new non-steroidal antiandrogen drug (NSAAD) that can bind directly to the ligand binding domain of the androgen receptor (AR), thereby inhibiting the AR nuclear translocation, further inhibiting DNA binding, and blocking AR-mediated transcription. ${ }^{10}$ The AR affinity of apalutamide is 7-10 times higher than that of bicalutamide. In a multicenter, double-blind, placebo-controlled, Phase III clinical trial (the SPARTAN [Selective Prostate Androgen 
Receptor Targeting with ARN-509] study ${ }^{11}$ ), 1207 M0 CRPC patients with a PSA-DT $\leq 10$ months were randomly divided into 2 groups, one receiving apalutamide plus endocrine therapy and the other placebo plus endocrine therapy. The results showed that the median metastasis-free survival (MFS) in the apalutamide group was 40.5 months, while in the placebo group it was 16.2 months $(\mathrm{HR}=0.28 ; P<0.001)$. Apalutamide decreased the risk of distal metastasis and death by $72 \%$. However, the survival benefit data was not fully reported. As regards the secondary endpoints of this study, the time to progression of symptoms $(\mathrm{HR}=0.45 ; P<0.001)$ and time to metastasis $(\mathrm{HR}=0.27 ; P<0.001)$ were both longer in the apalutamide group. The median progressionfree survival (PFS) for the apalutamide group was 40.5 months and 14.7 months for the placebo group $(\mathrm{HR}=0.29 ; P<0.001)$.

The most common adverse effects associated with apalutamide were weakness, high blood pressure, and rashes. In patients with a history of hypothyroidism, it is recommended that the thyroxine level is monitored during treatment.

\section{Enzalutamide}

Enzalutamide is a novel androgen receptor (AR) signal inhibitor, which competitively inhibits the binding of androgen to its receptor, thereby inhibiting AR nuclear transport, DNA binding, and the recruitment of coactivators. $^{12}$ The AR affinity of enzalutamide is 5-8 times higher than that of bicalutamide.

A multicenter, randomized, placebo-controlled, phase III clinical trial (the PROSPER [Safety and Efficacy Study of Enzalutamide in Patients With Nonmetastatic CastrationResistant Prostate Cancer] study ${ }^{13}$ ) involved 1401 patients with NM-CRPC who were randomly divided into two groups: enzalutamide combined with ADT, or placebo combined with ADT. The results showed that enzalutamide + ADT significantly prolonged the median metastasis-free survival in comparison with placebo + ADT (36.6 vs 14.7 months, respectively; HR $=0.29 ; P<0.001$ ) and the PSA progression time (37.2 vs 3.9 months, respectively; $\mathrm{HR}=0.07, P<0.001)$. However, no significant difference in overall survival (OS) was found between the two groups in a mid-term analysis.

Another phase III randomized, controlled trial (the STRIVE [Safety and Efficacy Study of Enzalutamide Versus Bicalutamide in Men with Prostate Cancer] study ${ }^{14}$ ) compared enzalutamide and bicalutamide in 139 patients with NMCRPC. At the time of publication of the study results, enzalutamide had achieved a PFS benefit in the NM-CRPC patients, as the median PFS with enzalutamide was longer than with bicalutamide (19.4 months vs 5.7 months, respectively). The proportions of patients in whom the PSA level decreased by more than $50 \%$ and $90 \%$ were also significantly higher in the enzalutamide group, and there was lower risk of radiological progression or death in this group. OS results, however, were not reported. The most common adverse events in the enzalutamide group were fatigue, back pain, hot flashes, falls, hypertension, dizziness, and loss of appetite.

Based on the above results, enzalutamide has been approved as one of the standard treatments for NMCRPC in Europe and the United States, but the drug has not yet been approved in China and its appropriate indications are still being evaluated.

\section{Abiraterone Acetate}

As both apalutamide and enzalutamide delay tumor progression in patients with NM-CRPC by further inhibiting androgen-signaling pathways, in theory, the use of abiraterone would have a similar effect. Abiraterone in combination with prednisone has shown survival benefits in patients with NM-CRPC and high-risk castrationsensitive prostate cancer. In a one-arm clinical trial (the IMAAGEN [Impact of Abiraterone Acetate on Prostate Specific AntiGEN] study ${ }^{15}$ ), 131 patients with NMCRPC were treated with abiraterone combined with prednisone, and the proportions of patients in whom the PSA level decreased by more than $50 \%$ and $90 \%$ were $86.9 \%$ and $59.8 \%$, respectively. At the time the results were published, the median time to PSA progression was 28.7 months and the median time to radiological progression had not yet been reached; therefore, there is currently no high-level evidence to sustain the long-term benefits of abiraterone. ${ }^{16}$ Recommendations for Treatment of NonMetastatic CRPC, see Box 2.

\section{Box 2 Treatment of Non-Metastatic CRPC (NM-CRPC)}

Recommendations
During the treatment of patients with NM-CRPC, there is a need to
achieve a balance between therapeutic benefits and drug toxicity.
Taking into account the efficacy, safety and accessibility of drugs in
China, the optimal treatment for NM-CRPC patients with a high risk
of metastasis (PSADT < 10 months) is a combination of apalutamide
and ADT. Enzalutamide is also one of the standard regimens for NM-
CRPC treatment in countries and regions where it is approved
(Evidence level A). It should be noted that the imaging modality is
traditional imaging (such as bone scans, CT, etc.).

(Continued) 
Box 2 (Continued).

Recommendation I: Optimal treatment: The addition of apalutamide $240 \mathrm{mg} /$ day to ADT (Evidence level A).

Recommendation 2: Optional treatment I: If the patient is unwilling to receive the optimal regimen or the optimal regimen cannot be administered on time, continuing ADT with close monitoring is recommended (Evidence level $\mathrm{C}$ )

Recommendation 3: Optional treatment 2: If the risk of metastasis is high and patients are unwilling to be observed only, abiraterone acetate plus prednisolone could be prescribed (Evidence level C).

Recommendation 4: Except for clinical trials, systemic

chemotherapy or immunotherapy agents are not recommended for patients with NM-CRPC.

Recommendation 5: Denosumab slightly delays bone metastasis (by about 3 months), ${ }^{17}$ but has no advantage over other drugs in improving overall survival or quality-of-life, and it is not recommended for patients with NM-CRPC.

Recommendation 6: There is no evidence of survival benefit for first-generation antiandrogenic drugs in patients with NM-CRPC, and they are no longer recommended on the premise that drugs with definite efficacy are available.

Recommendation 7: Patients who do not accept the above treatment regimens may choose to participate in clinical trials.

\section{Localized Clinically Progressive NM-CRPC}

If the patient has not previously received any type of treatment, radiotherapy is recommended as first or salvage surgery in these patients. ${ }^{9}$

\section{Clinical Management of Metastatic CRPC}

Asymptomatic/minimally symptomatic patients in good physical condition, with no prior history of chemotherapy

\section{Definition}

M1 CRPC patients in good physical condition who are asymptomatic or minimally symptomatic are patients with metastatic lesions but no pain or only mild pain. Mild pain is defined as pain that can be relieved by non-steroidal anti-inflammatory drugs (NSAIDs) or analgesics such as acetaminophen.

\section{Optimal Treatment}

\section{Novel Endocrine Therapy}

Abiraterone Combined with Prednisone

In a phase III randomized, placebo-controlled clinical trial (the COU-AA-302 [NCT00887198] study $^{18}$ ), 1088 patients with mCRPC who had not previously received chemotherapy were randomly divided into two groups: abiraterone plus prednisone or placebo plus prednisone. The results showed that abiraterone significantly prolonged radiological progression-free survival (16.5 vs 8.2 months, respectively; HR = $0.52 ; P<0.001$ ), and improved OS by 4.4 months ( 34.7 vs 30.3 months, respectively; $\mathrm{HR}=0.81 ; P=0.0033$ ). In addition, abiraterone delayed the progression of pain, delayed the use of chemotherapy and opioids, and hence delayed deterioration of the patients' physical condition. Although grade 3-4 corticosteroid-related adverse events and liver dysfunction were more common in the abiraterone + prednisone group, most of these events were mild, and the overall tolerance of the regimen was good. ${ }^{19,20}$

\section{Enzalutamide}

A double-blind, phase III trial (the PREVAIL [Enzalutamide in men with chemotherapy-naive metastatic prostate cancer; NCT01212991] study ${ }^{21,22}$ ) compared enzalutamide and placebo in 1717 asymptomatic or mildly symptomatic patients who had not received chemotherapy or were previously treated with abiraterone. The results showed that compared with the placebo group, enzalutamide (160 $\mathrm{mg}$ once daily) significantly reduced the risk of radiological progression $(\mathrm{HR}=0.19$; $P<0.001)$ and mortality $(\mathrm{HR}=0.71 ; P<0.001)$, thus delaying the use of chemotherapy and the occurrence of bone-related events. Enzalutamide prolonged the time to PSA progression, increased the response rate of soft tissue lesions, and increased the proportion of patients whose PSA decreased by more than $50 \%$. Enzalutamide also slowed pain progression, delaying the first use of opioids and deterioration of patients' physical condition. The most common adverse events of enzalutamide included fatigue and hypertension.

Enzalutamide (160 $\mathrm{mg}$ once daily) is the preferred treatment option in countries and regions where it is available (Evidence level A).

\section{Chemotherapy}

Docetaxel promotes apoptosis of cancer cells by inhibiting microtubule depolymerization and weakening the expression of Bcl-2 and Bcl-xL genes. ${ }^{23}$ Its most common adverse effects are bone marrow suppression, fatigue, alopecia, diarrhea, neuropathy and angioneurotic edema. In comparison with mitoxantrone, the administration of docetaxel every three weeks significantly prolonged the median survival time (18.9 vs 16.5 months, respectively), reduced the risk of death $(\mathrm{HR}=0.75 ; P=0.009)$, and improved pain symptoms and quality-of-life. ${ }^{24}$ However, symptomatic patients 
were also included in this cohort study, and docetaxel treatment was associated with a higher incidence of severe adverse events and drug withdrawal rates.

\section{Optional Treatments}

If the patient cannot tolerate abiraterone and has no indication for chemotherapy, conventional second-line endocrine therapy or immunotherapy can be considered. Simple castration combined with first-generation antiandrogenic drugs or corticosteroids can cause a transient decrease in PSA (in 30\% of patients), but the duration of PSA remission is relatively short and there is no long-term survival benefit. Patients who have received combined androgen blocking therapy may consider suspending the use of antiandrogen drugs while observing the related withdrawal reactions and manifestations. Nevertheless, although the weekly administration of mitoxantrone plus prednisone does not benefit overall survival, it can control and alleviate the disease and improve the quality-of-life of patients. $^{25}$ Sipuleucel-T is an autologous cell immunotherapy agent, that is currently approved only in the United States. The phase III IMPACT study ${ }^{26}$ showed that compared with placebo, sipuleucel-T reduced the risk of death by $22 \%(P=0.03)$ and prolonged the median survival time (25.8 vs 21.7 months, respectively); it was generally well tolerated, and its most common adverse effects included chills, fever and headaches, which were usually temporary. Recommendations, see Box 3.

Box 3 Treatment of Asymptomatic or Minimally Symptomatic mCRPC Patients, in Good Physical Condition and Without a Prior History of Chemotherapy

\section{Recommendations}

Recommendation I: When choosing the treatment plan, firstly consider the least toxic drug available, and formulate the medication plan after fully communicating with patients, taking into account factors such as the clinical status, convenience of administration and patient preference.

Recommendation 2: Patients with mCRPC should continue ADT treatment (Evidence level B).

Recommendation 3: Optimal treatment I (based on androgen deprivation therapy): combination treatment with abiraterone $1000 \mathrm{mg}$ (once daily) and prednisone $5 \mathrm{mg}$ (twice daily) [Evidence level A].

Recommendation 4: Optimal treatment 2: If the disease is aggressive, docetaxel $75 \mathrm{mg} / \mathrm{m}^{2}$ (once every 3 weeks) combined with prednisone $5 \mathrm{mg}$ (twice daily) could be considered (Evidence level B).
}

Box 3 (Continued).

Recommendation 5: Optional treatment: For patients who cannot tolerate abiraterone, have no indication for chemotherapy, and have previously only received castration treatment, first-generation antiandrogens or corticosteroids are recommended (Evidence level C).

Recommendation 6: Optional treatment: For patients who cannot tolerate abiraterone, have no indication for chemotherapy, and have previously received combined androgen blocking therapy, it is recommended that the use of antiandrogen drugs be suspended while observing the related withdrawal reactions and manifestations (Evidence grade C). ${ }^{27-29}$

Recommendation 7: Optional treatment: mitoxantrone + prednisone (Evidence grade B).

Recommendation 8: Patients who do not accept the above treatment regimens may choose to participate in clinical trials.

\section{Symptomatic mCRPC Patients in Good Physical Condition Without a Prior History of Chemotherapy Definition, Monitoring and Management}

In mCRPC patients with significant pain symptoms, it should firstly be considered that the pain symptoms in these patients are a result of prostate cancer metastasis, rather than other diseases. Eventually, significant pain requires the regular use of opioids for pain management. Symptomatic CRPC M1 patients should be followed-up every 3 months, and should be clinically re-evaluated when the PSA level doubles or new symptoms occur.

\section{Optimal Treatment}

\section{Abiraterone}

In the COU-AA-302 study, abiraterone combined with prednisone significantly prolonged overall survival. ${ }^{18}$ Although this study did not include mCRPC patients with significant pain symptoms, it is feasible to use this treatment in symptomatic mCRPC patients, taking into consideration the mechanism of action of abiraterone and its significant pain-relieving and bone benefits.

\section{Enzalutamide}

Enzalutamide (160 mg once daily) is the preferred option in countries and regions where this drug is available (Evidence level A). 


\section{Chemotherapy (Docetaxel)}

A 3-week docetaxel regimen combined with prednisone can significantly improve the bone pain response rate $(35 \%$ vs $22 \% ; P=0.01$ ), improve health-related quality-of-life, and improve survival compared with mitoxantrone plus prednisone. ${ }^{24}$ The results of a study by Chinese investigators showed that the PSA response rate in the docetaxel group was $70 \%$; the response was sustained for 5.6 months, and the patients' median survival time was 27.8 months. ${ }^{30}$ A 2-week docetaxel regimen provides better control, improves the time to disease progression and the PSA decline rate, and has good patient tolerance. Hence, the 2-week docetaxel regimen can be used as an alternative regimen in patients with poor tolerance of docetaxel. ${ }^{31}$

\section{Optional Treatments} Mitoxantrone

Mitoxantrone is a topoisomerase II inhibitor that interferes with DNA replication, transcription and repair in cancer cells. Its common adverse effects include nausea, vomiting, alopecia, cardiotoxicity and bone marrow suppression, with bone marrow suppression and cardiotoxicity being delayed effects. The most serious adverse effect is irreversible cardiomyopathy and it may cause heart failure. ${ }^{32}$ Mitoxantrone is mainly used for palliative chemotherapy in patients with symptomatic mCRPC in poor physical condition (ie in the event of docetaxel intolerance).

\section{Radionuclide Therapy}

Systemic radionuclide therapy with samarium-153 or strontium-89 is usually indicated in patients who do not respond to palliative chemotherapy and who are not suitable for local external radiotherapy. ${ }^{33,34}$ Prostate cancer patients with extensive bone metastasis can sometimes benefit from this treatment method, but bone marrow suppression should be considered when applying it.

\section{Radium-223}

Radium-223 is currently the only bone-specific drug proven to prolong overall survival. ${ }^{35}$ It can emit high-energy $\alpha$-rays which cause the breakage of double-stranded DNA in tumor cells at bone metastasis sites.

The phase III ALSYMPCA (ALpharadin in SYMPtomatic Prostate CAncer) trial $^{36}$ showed that radium-233 improved overall survival in progressive mCRPC patients without visceral metastasis in comparison with placebo (median OS of 14.9 vs 11.3 months, respectively; $\mathrm{HR}=0.695 ; P=0.00007)$, and delayed the onset of first bone-related events (15.6 vs 9.8 months, respectively; $\mathrm{HR}=0.658 ; P=0.00037)$, thereby significantly improving the patients' health-related quality-of-life. Radium-223 was effective and safe in both patients who had and had not previously been treated with docetaxel. ${ }^{37}$

\section{Symptomatic mCRPC Patients with Significant Pain Symptoms and Good Physical Condition, Without a Prior History of Chemotherapy Recommendations, Box 4}

Box 4 Treatment of Symptomatic mCRPC Patients with Significant Pain Symptoms Who are in Good Physical Condition and Without a Prior History of Chemotherapy

\footnotetext{
Recommendations

Recommendation I: Optimal treatment: $1000 \mathrm{mg}$ abiraterone (once daily) combined with $5 \mathrm{mg}$ prednisone (twice daily) [Evidence level A] Recommendation 2: Optimal treatment: Docetaxel $75 \mathrm{mg} / \mathrm{m}^{2}$ (once every 3 weeks) combined with prednisone $5 \mathrm{mg}$ (twice daily) [Evidence level A).

Recommendation 3: Optional treatment: Mitoxantrone (Evidence level B) or radionuclide therapy (Evidence level C).

Recommendation 4: Optional treatment: Radium-233 can be used for patients without visceral or bone metastasis (Evidence grade B).

Recommendation 5: Patients who do not accept the above treatment regimen may choose to participate in clinical trials.
}

\section{mCRPC with Progression After Chemotherapy}

Some patients with mCRPC who receive docetaxel chemotherapy in the early stages of metastasis experience disease progression during the course of this treatment. But as they are still in good physical condition, the focus of treatment for these patients should be to maintain their relatively good health and avoid excessive toxicity and adverse effects. The benefits of novel endocrine therapy are comparable, if not better than that of the second-line intravenous chemotherapy. The new taxane chemotherapy agent cabazitaxel can be used in patients with mCRPC who have a poor response to docetaxel chemotherapy, but as cabazitaxel is intravenously administered, the level of toxicity is significant and clinical benefit is rather limited. Therefore, novel endocrine therapy should be given priority in the treatment of mCRPC. 


\section{Novel endocrine therapy}

Abiraterone Combined with Prednisone

The phase III COU-AA-301 trial ${ }^{38}$ included 1195 patients who had a poor response to docetaxel, and the final analysis showed that the combination of abiraterone and prednisone significantly prolonged OS by 4.6 months compared with placebo (15.8 vs 11.2 months, respectively; $\mathrm{HR}=0.74 ; P<0.001$ ), with all subgroups showing benefits. All other secondary endpoints also favored abiraterone: the median time to PSA progression was prolonged ( 8.5 vs 6.6 months, respectively), as was the radiological time to progression (5.6 vs 3.6 months), and the proportion of patients with a PSA decline of more than $50 \%$ was higher ( $29 \%$ vs $5.5 \%$ ). The most common adverse effects that led to drug discontinuation included elevated serum levels of aspartate and/or alanine aminotransferase, or heart disease. Therefore, during treatment with abiraterone combined with prednisone, liver function, blood potassium and phosphorus levels, and blood pressure should be monitored on a monthly basis in the initial stages of treatment, together with symptomatic assessment of cardiac diseases, especially for patients with a history of cardiovascular diseases.

\section{Enzalutamide}

In the phase III randomized, placebo-controlled AFFIRM (A Multinational Phase 3, Randomized, Double-blind, Placebocontrolled Efficacy And Safety Study of Oral Mdv3100 In Patients With Progressive Castration-resistant Prostate Cancer Previously Treated with Docetaxel Based Chemotherapy, NCT00974311) trial, ${ }^{39} 1199$ patients were randomly divided 2:1 into two groups, enzalutamide or placebo, and all still continued to receive ADT treatment. Enzalutamide + ADT treatment prolonged overall survival in comparison with placebo + ADT treatment (18.4 vs 13.6 months, respectively; HR $=0.63 ; P<0.001$ ), and other secondary endpoints were also significantly in favor of the enzalutamide group, including the proportion of patients with PSA declines of more than 50\%, radiological remission, radiological progression-free survival, time to skeletal-related events (SREs), and an improved quality-of-life. The main toxicities were fatigue, diarrhea, hot flashes, headache and seizures (incidence $0.6 \%$ vs $0 \%$ in the placebo group). A final long-term follow-up analysis of this trial confirmed an overall survival benefit. ${ }^{22}$

\section{Chemotherapy}

\section{Cabazitaxel}

Cabazitaxel is a semi-synthetic taxane derivative that has been used in patients with mCRPC who failed to respond to docetaxel chemotherapy. In an international phase III randomized clinical trial (TROPIC [XRP6258 Plus Prednisone Compared to Mitoxantrone Plus Prednisone in Hormone Refractory Metastatic Prostate Cancer] study ${ }^{40}$ ), 755 patients with progressive mCRPC were randomly divided into two groups: one group was treated with $25 \mathrm{mg} / \mathrm{m}^{2}$ intravenous cabazitaxel plus prednisone, while the other group received $12 \mathrm{mg} / \mathrm{m}^{2}$ mitoxantrone plus prednisone. Compared with the mitoxantrone group, OS in the cabazitaxel group was prolonged by 2.4 months (15.1 vs 12.7 months, respectively; $\mathrm{HR}=0.72 ; P<$ $0.0001)$, and the PFS was also significantly improved ( $2.8 \mathrm{vs}$ 1.4 months; $P<0.0001)$. The main toxicity of cabazitaxel is bone marrow suppression. Other common adverse effects include diarrhea, nausea, vomiting, and fatigue. Postapproval studies found that the OS of patients treated with a $20 \mathrm{mg} / \mathrm{m}^{2}$ dose of cabazitaxel was not inferior to that of patients treated with a $25 \mathrm{mg} / \mathrm{m}^{2}$ dose, but the toxicity was lower in second-line treatment. Therefore, low-dose administration $^{41,42}$ should be preferred.

\section{mCRPC Patients in Good Physical Condition Presenting with Progression After Chemotherapy Recommendations, Box 5}

Box 5 Treatment of mCRPC Patients in Good Physical Condition Presenting with Progression After Chemotherapy

\section{Recommendations}

Recommendation I: Optimal treatment: $1000 \mathrm{mg}$ of abiraterone (once daily) combined with $5 \mathrm{mg}$ prednisone (twice daily) [Evidence level A].

Recommendation 2: Optional treatment: Patients without visceral metastasis, in good physical condition and with obvious symptoms of bone metastasis can be treated with radium-233 (Evidence grade B).

Recommendation 3: Mitoxantrone combined with prednisone can relieve pain symptoms caused by prostate cancer (Evidence level B).

Recommendation 4: Patients who do not accept the above treatment regimens may choose to participate in clinical trials.

\section{Symptomatic mCRPC Patients in Poor Physical Condition}

Patients with poor physical fitness (Eastern Cooperative Oncology Group score 3-4) are generally not included in clinical trials, and when it comes to treatment options, it is recommended to refer to patients in good physical condition. The poor physical condition of some patients is directly related to the progression of cancer. However, effective anticancer 
therapy may restore physical fitness. Clinicians need to fully communicate with patients and assess the risks and benefits of treatment in comparison with previous medications, especially considering the health-related quality-of-life of patients. The clinical studies of abiraterone and enzalutamide only included patients in good physical condition, but the tolerance of these drugs in patients with poor physical condition was better than that of chemotherapy, and they are therefore a more reasonable choice. Recommendations, see Box 6.

Box 6 Treatment of Symptomatic mCRPC Patients in Poor Physical Condition with and Without a Prior History of Chemotherapy

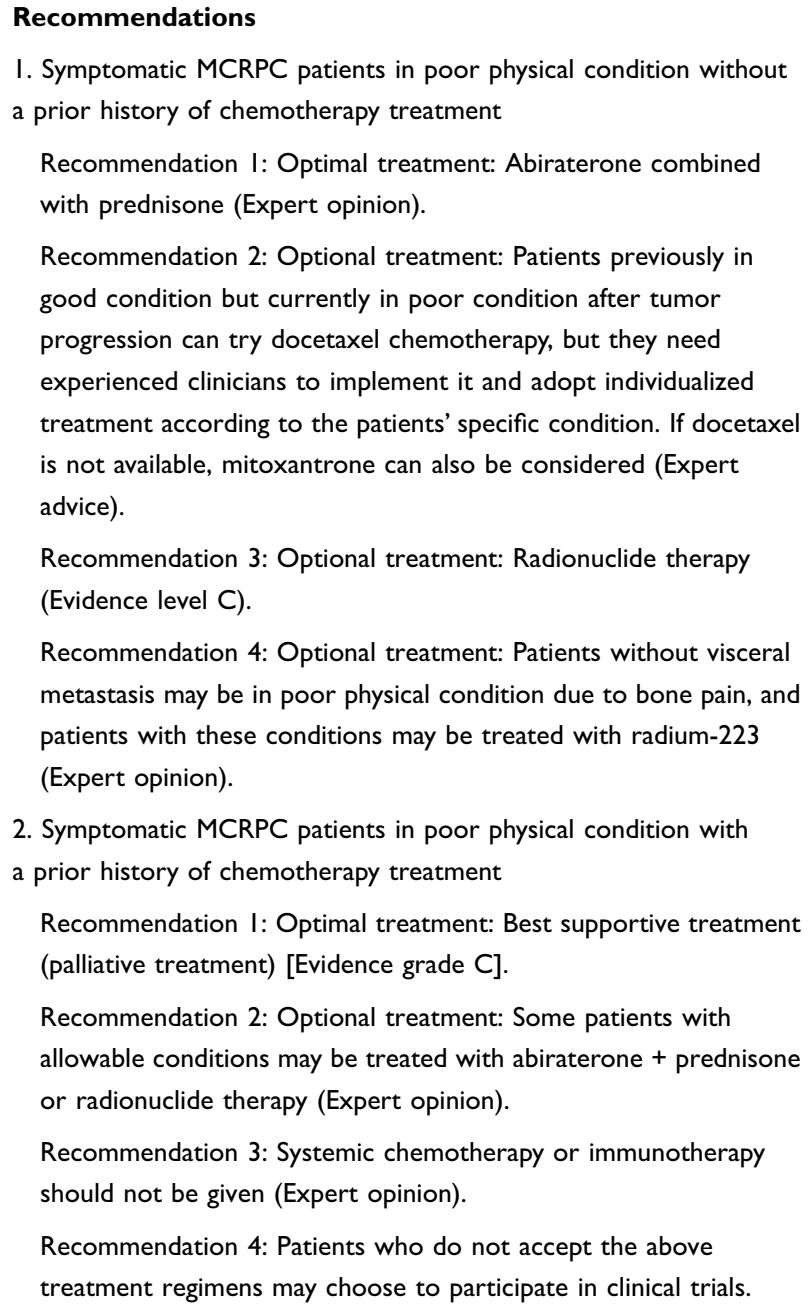

2. Symptomatic MCRPC patients in poor physical condition with a prior history of chemotherapy treatment

Recommendation I: Optimal treatment: Best supportive treatment (palliative treatment) [Evidence grade C].

Recommendation 2: Optional treatment: Some patients with allowable conditions may be treated with abiraterone + prednisone or radionuclide therapy (Expert opinion).

Recommendation 3: Systemic chemotherapy or immunotherapy should not be given (Expert opinion).

Recommendation 4: Patients who do not accept the above treatment regimens may choose to participate in clinical trials.

\section{Specific Pathological Types of CRPC}

De novo small cell carcinoma in untreated prostate cancers occurs rarely and is very aggressive. ${ }^{43}$ Treatment-associated small cell/neuroendocrine prostate cancer that occurs in men with metastatic CRPC is more common. ${ }^{44}$ A poor response to first-line androgen deprivation therapy by patients and clinical or radiological disease progression, but no increase in the PSA level may due to the pathological type of cancer such as prostate cancer with neuroendocrine differentiation or small cell carcinoma. Puncture biopsy may be considered to obtain a definite diagnosis. These patients can be treated with combination chemotherapy, such as cisplatin combined with etoposide, or carboplatin combined with etoposide. ${ }^{45}$

\section{Therapeutic Response Evaluation of CRPC Treatment}

An adequate drug exposure time is critical for evaluating the efficacy of cancer therapy. CRPC is the final stage of prostate cancer with high heterogeneity, and few alternative therapies are available in China. It is recommended that patients receive first-line drug therapies with evidence of life benefit, and the lowest drug exposure time to evaluate their efficacy is 12 weeks.

The efficacy evaluation of CRPC drug therapy needs to be combined with three aspects: PSA levels, radiological detection of soft tissue and bone metastases, and clinical symptoms. The St. Gallen Consensus ${ }^{46}$ and the PCWG3 guidelines $^{47}$ of the 2015 edition of Advanced Prostate Cancer, suggest that two of the three indicators: PSA, radiological findings, and clinical symptoms, can be used to judge disease progression. Because of the phenomenon of "scintillation", we should avoid relying solely on changes of PSA levels or bone scans to judge the curative effect. Evidence of disease progression is an important factor to consider when replacing therapeutic regimens, but it should be judged comprehensively together with consideration of

Box 7 Therapeutic Response Evaluation of CRPC

\section{Recommendations}

Recommendation I: The minimum drug exposure time for evaluating the efficacy of the first-line treatment regimen is 12 weeks (Evidence level C).

Recommendation 2: The criteria for judging disease progression are: the PSA level, radiological detection of metastases, and clinical symptoms (Evidence level B).

Recommendation 3: A comprehensive decision-making process should be applied to change the treatment plan according to the progress of the disease and the patient's condition (Evidence level C).

whether second-line drugs are better than existing regimens. Recommendations for Therapeutic Response Evaluation of CRPC, see Box 7. 


\section{Bone Health Related Therapy}

Prostate cancer patients with metastasis have a higher risk of bone complications, mainly due to the physiological decrease of bone mineral density associated with treatment and bone metastasis of tumors. Treatments for bone health can prevent or delay the occurrence of bone-related events.

\section{Zoledronic Acid}

Bisphosphonates are potent inhibitors of bone absorption that block pathological osteolysis by inhibiting the activation and function of osteoclasts. However, apart from zoledronic acid, other bisphosphonates do not show beneficial effects in patients with mCRPC. Compared with placebo, CRPC patients treated with zoledronic acid have fewer bone-related events (38\% vs 49\%, respectively) and a longer time to first skeletal-related events (SREs) [488 vs 321 days, respectively], but with no significant difference in OS. ${ }^{48,49}$ It should be noted that zoledronic acid cannot be used in patients whose basal creatinine clearance rate is less than $30 \mathrm{~mL} / \mathrm{min}$.

\section{Denosumab}

RANK ligand (RANK-L) is an important driver of osteoclast function and survival. Denosumab is a humanized monoclonal antibody against RANK-L that can inhibit osteoclast-mediated bone destruction and bone metastasis. In patients with mCRPC, denosumab significantly delayed the first SRE in comparison with zoledronic acid by 3.6 months (20.7 vs 17.1 months, respectively; HR 0.82 ; validity $P=0.008$ ), but the absolute incidence of SREs was similar in both groups. ${ }^{50}$ There is no need to adjust the dosage according to renal function when using denosumab, but there is no study on the application of this drug in patients with basic creatinine clearance rates less than $30 \mathrm{~mL} / \mathrm{min}$.

The treatment-related toxicities of zoledronic acid and denosumab are similar, including hypocalcemia (13\% vs $6 \%$, respectively), joint pain, and jaw necrosis (1-2\%). The risk of mandibular necrosis should be addressed in patients who have been treated with denosumab or

Box 8 Bone-Related Therapy in mCRPC Patients

\footnotetext{
Recommendations

Recommendation I: Clinicians should provide prophylactic treatment (such as calcium and vitamin D supplementation) for fractures and bone-related events (Evidence level C).

Recommendation 2: Patients with MCRPC may be treated with either $120 \mathrm{mg}$ of denosumab or $4 \mathrm{mg}$ of zoledronic acid every 4 weeks to prevent bone-related events (Evidence level C).
}

zoledronic acid for more than two years. Recommendations for Bone-Related Therapy in mCRPC Patients, see Box 8.

\section{Other Supportive Treatment}

Bone metastases of prostate cancer are mostly sensitive to radiotherapy. External radiation therapy for specific lesions can partially or completely relieve pain in most patients. $^{51}$ For CRPC patients with multiple bone metastases, systemic strontium-89 radionuclide therapy may be effective in relieving symptoms, but there is a risk of severe bone marrow suppression and blood transfusion dependence. ${ }^{52-54}$ Samarium-153 can also alleviate pain in patients with bone metastasis. ${ }^{55-57}$

When spinal cord compression is suspected, immediate diagnosis and treatment are needed. Therapeutic options include surgery plus radiotherapy, internal fixation plus radiotherapy, or radiotherapy plus high-dose corticosteroids. ${ }^{58}$

\section{Oligometastatic mCRPC}

Oligometastasis refers to patients with $\leq 5$ lymph nodes and/or bone metastatic lesions. Oligometastatic prostate cancer patients have a better prognosis and longer survival than those with extensive metastasis, ${ }^{59,60}$ but overall, oligometastatic prostate cancer is still considered as a systemic disease. Local therapy can alleviate the symptoms of oligometastatic CRPC patients, delay systemic therapy, and prolong OS and/or PFS. Recommendations for Treatment of Oligometastatic CRPC, see Box 9.

\section{Box 9 Treatment of Oligometastatic CRPC}

\section{Recommendation \\ Recommendation I: Novel endocrine therapy for oligometastatic CRPC patients (abiraterone, enzalutamide) and local ablation therapy are recommended. Tumor reduction surgery, prostate cancer resection surgery, or radiotherapy in patients with corresponding favorable conditions (Expert opinion).}

\section{Other Novel Drugs}

If the long-term prognosis of CRPC is not good, patients should be advised to actively participate in clinical trials of new drugs. At present, there are many investigations of the significance of novel endocrine drugs, different targeted therapy drugs, and immunotherapy agents in the treatment of prostate cancer (eg of darolutamide, bevacizumab, 
cilengitide, the PARP inhibitors oliparib and niraparib, and pembrolizumab).

\section{CRPC Genetic Monitoring}

With the wider application of next-generation sequencing (NGS) for the clinical diagnosis and treatment of tumors, including prostate cancer, more and more patients have benefited from an accurate diagnostic and treatment strategy of prostate cancer. Gene detection is helpful for evaluating the prognosis of patients more accurately and stratifying the risk of disease progression; suggesting the sensitivity of specific drugs, guiding precise treatment, and improving the prognosis of patients; for accumulating data to improve the gene mutation spectrum and related characteristics of prostate cancer patients in China; further understanding the molecular mechanisms related to disease progression, metastasis, recurrence, and the efficacy of CRPC treatment; and for assisting the development of new drugs. However, how to use the precise localization aspect of NGS to benefit patients with prostate cancer while avoiding over-detection, further interpretation of detection results, and formulating individualized precise treatment plans are important issues for clinicians.

Therefore, it is suggested that patients with confirmed mCRPC, regardless of family history, should undergo gene mutation detection after first-line treatment. In addition to mCRPC patients, other types of prostate cancer patients for whom gene detection is recommended include: ${ }^{61}$ (1) patients with confirmed metastatic prostate cancer; (2) prostate cancer patients with pancreatic cancer, prostate cancer patients with a direct relative who has ovarian cancer, or a direct relative with breast cancer at an age of less than 50 years or 2 relatives with breast cancer, or patients at any age with a direct relative who has prostate cancer of any grade (all ages, Gleason score $\geq 7$ points); (3) 2 or more prostate cancer patients with a suspected family history of hereditary ovarian cancer-breast cancer syndrome, hereditary prostate cancer syndrome, and Lynch syndrome; and (4) patients with a history of pancreatic cancer, whose cancer tissue tests detected mutations in genes associated with a cancer risk but lacked embryonic line validation. The detected genes are as follows: embryonic and somatic gene mutations of DNA, homologous recombinant repair genes such as BRCA1/2, ATM, PALB 2 and FANCA; MMR genes (MLH1, MSH2, PMS2, MSH6 and EPCAM); and HOXB13 genes of familial hereditary prostate cancer patients. Wei et al observed similar germline DNA repair genes mutation frequencies,
Box 10 mCRPC Genetic Monitoring

\section{Recommendations}

Recommendation I: It is recommended that patients with confirmed mCRPC, with or without a family history, undergo gene mutation testing after disease progression following the first-line treatment. It is recommended to test for genetic mutations in BRCAI/2, ATM, PALB 2 and FANCA homologous recombinant repair genes in order to guide the use of platinum drugs and participate in clinical trials (including trials of PARP inhibitors); and MMR genes (MLHI, MSH2, PMS2, MSH6 and EPCAM) [Evidence level B].

Recommendation 2: In prostate cancer patients with a family history of BRCAI/2 gene-related tumors or patients with family members carrying BRCAI/2 and MMR gene mutations, or a finding of cancer cell detection mutations related to gene therapy, it is recommended to investigate for embryonic BRCAI/2 and MMR genes (MLHI, MSH2, PMS2, MSH6 and EPCAM) [Evidence level B].

Recommendation 3: In addition, after evaluating and analyzing the family history of prostate cancer, $\mathrm{HOXBI} 3$ mutations can be investigated (Evidence grade $\mathrm{C}$ ).

although there is a large disparity in the risk of prostate cancer between China and western countries. Therefore, given the ethnic diversity, we advocate a comprehensive analysis of detailed mutation spectra to refine management strategies further. ${ }^{62}$ Recommendations for mCRPC Genetic Monitoring, see Box 10.

\section{Recommendation for Multidisciplinary Approach for CRPC} CRPC is a disease with a high rate of heterogeneity, and as a result, individual cases are very complicated. The conventional "one on one" mode of therapy cannot fulfil the requirements and solve all the problems of optimal treatment. Hence, we highly recommend a multidisciplinary team (MDT) treatment approach which involves a relatively fixed panel of clinical specialists coming together and discussing patients with specific diseases and recommending the personalized, optimal, and best suited treatment plan for the patient.

Based on the experience of 100 hospitals and more than 100 online and offline MDT consultation platforms for urinary system cancer in China, it is recommended that the organizational structure of the MDT includes five parts: ${ }^{63}$ (1) the convenor (Chief Expert), who is generally held to be an authoritative urological expert; (2) experts in urology, radiotherapy, oncology, pathology, radiodiagnostics, nuclear medicine, ultrasonography, and interventional medicine who should generally be qualified as senior attending physicians 
and have experience in the diagnosis and treatment of CRPC or prostate cancer, with special physicians invited to participate in relevant departments as required; (3) recorders; (4) consultation secretaries; and (5) medical institution personnel.

A complete MDT standardization procedure consists of 12 steps: appointment, case preparation, condition report, image analysis, expert discussion, decision-making, patient and family meetings, discussion records, program implementation, monitoring and evaluation, program revision, and follow-up.

\section{Acknowledgments}

Members of the Expert Group are: Songzhi He, Zhaohong He, Quanzhi Hu, Xiang Li, Hong Luo, Tiejun Pan, Jun Qi, Benkang Shi, Quanzhong Sun, Jinchun Xing, Yong Xu, Shaoxing Zhu, Qing Zou.

\section{Disclosure}

The authors report no conflicts of interest in this work.

\section{References}

1. China Anti-Cancer Association Genitourinary Cancer Committee. Expert consensus on diagnosis and treatment of castration-resistant prostate cancer in China. Zhejiang Med. 2016;38(14):1133-1145.

2. Andrews J, Guyatt G, Oxman AD, et al. GRADE guidelines: 14 . Going from evidence to recommendations: the significance and presentation of recommendations. J Clin Epidemiol. 2013;66:719-725. doi:10.1016/j.jclinepi.2012.03.013

3. Mottet N, van den Bergh RCN, Briers E, et al. EAU-ESTRO-ESURSIOG guidelines on prostate cancer. Presented at the EAU Annual Congress, Copenhagen; 2018.

4. Bray F, Ferlay J, Soerjomataram I, et al. Global cancer statistics 2018: GLOBOCAN estimates of incidence and mortality worldwide for 36 cancers in 185 countries. CA Cancer J Clin. 2018;68:394-424. doi:10.3322/caac.v68.6

5. Chen W, Zheng R, Baade PD, et al. Cancer statistics in China, 2015. CA Cancer J Clin. 2016;66(2):115-132. doi:10.3322/caac.21338

6. Smith MR, Kabbinavar F, Saad F, et al. Natural history of rising serum prostate-specific antigen in men with castrate nonmetastatic prostate cancer. J Clin Oncol. 2005;23:2918-2925. doi:10.1200/ JCO.2005.01.529

7. Smith MR, Cook R, Lee K-A, et al. Disease and host characteristics as predictors of time to first bone metastasis and death in men with progressive castration-resistant nonmetastatic prostate cancer. Cancer. 2011;117:2077-2085. doi:10.1002/cncr.25762

8. Crawford ED, Stone NN, Yu EY, et al. Challenges and recommendations for early identification of metastatic disease in prostate cancer. Urology. 2014;83(3):664-669. doi:10.1016/j.urology.2013.10.026

9. Gómez Caamaño A, González San Segundo C, Henríquez I, et al. Consensus on management of castration resistant prostate cancer on behalf of the Urological Tumours 9 Working Group (URONCOR) of the Spanish Society of Radiation Oncology. Clin Transl Oncol. 2019;21(4):420-432. doi:10.1007/s12094-018-1940-2

10. Clegg NJ, Wongvipatt J, Joseph JD, et al. ARN-509: a novel antiandrogen for prostate cancer treatment. Cancer Res. 2012;72:1494-1503. doi:10.1158/0008-5472.CAN-11-3948
11. Smith MR, Saad F, Chowdhury S, et al. Apalutamide treatment and metastasis-free survival in prostate cancer. $N$ Engl $J$ Med. 2018;378:1408-1418. doi:10.1056/NEJMoa1715546

12. Tran C, Ouk S, Clegg NJ, et al. Development of a second-generation antiandrogen for treatment of advanced prostate cancer. Science. 2009;324(5928):787-790. doi:10.1126/science.1168175

13. Hussain M, Fizazi K, Saad F, et al. Enzalutamide in men with non-metastatic, castration-resistant prostate cancer. $N$ Engl J Med. 2018;378(26):2465-2474. doi:10.1056/NEJMoa1800536

14. Penson DF, Armstrong AJ, Concepcion R, et al. Enzalutamide versus bicalutamide in castration-resistant prostate cancer: the STRIVE trial. J Clin Oncol. 2016;34:2098-2106. doi:10.1200/ JCO.2015.64.9285

15. Ryan CJ, Crawford ED, Shore ND, et al. The IMAAGEN study: effect of abiraterone acetate and prednisone on prostate specific antigen and radiographic disease progression in patients with nonmetastatic castration resistant prostate cancer. J Urol. 2018;200 (2):344-352. doi:10.1016/j.juro.2018.03.125

16. Cookson MS, Roth BJ, Dahm P, et al. Castration-Resistant Prostate Cancer. AUA Guideline; 2018.

17. Smith MR, Saad F, Coleman R, et al. Denosumab and bone-metastasis-free survival in men with castration-resistant prostate cancer: results of a global phase 3, randomised, placebo-controlled trial. Lancet. 2012;379:39-46. doi:10.1016/ S0140-6736(11)61226-9

18. Ryan CJ, Smith MR, Fizazi K, et al. Abiraterone acetate plus prednisone versus placebo plus prednisone in chemotherapy-naïve men with metastatic castration-resistant prostate cancer (COU-AA-302): final overall survival analysis of a randomised, double-blind, placebocontrolled phase 3 study. Lancet Oncol. 2015;16:152-160. doi:10.1016/S1470-2045(14)71205-7

19. Ryan CJ, Smith MR, de Bono JS, et al. Abiraterone in metastatic prostate cancer without previous chemotherapy. $N$ Engl $J$ Med. 2013;368:138. doi:10.1056/NEJMoa1209096

20. Rathkopf DE, Smith MR, de Bono JS, et al. Updated interim efficacy analysis and long-term safety of abiraterone acetate in metastatic castration-resistant prostate cancer patients without prior chemotherapy (COU-AA-302). Eur Urol. 2014;66(5):815-825. doi:10.1016/j. eururo.2014.02.056

21. Beer TM, Armstrong AJ, Rathkopf DE, et al. Enzalutamide in metastatic prostate cancer before chemotherapy. $N$ Engl $J$ Med. 2014;371:424-433. doi:10.1056/NEJMoa1405095

22. Beer TM, Armstrong AJ, Rathkopf D, et al. Enzalutamide in men with chemotherapy-naive metastatic castration-resistant prostate cancer: extended analysis of the phase 3 PREVAIL study. Eur Urol. 2017;71(2):151-154. doi:10.1016/j.eururo.2016.07.032

23. Pienta KJ. Preclinical mechanisms of action of docetaxel and docetaxel combinations in prostate cancer. Semin Oncol. 2001;28(4, Suppl 15):3-7. doi:10.1016/S0093-7754(01)90148-4

24. Tannock IF, de Wit R, Berry WR, et al. Docetaxel plus prednisone or mitoxantrone plus prednisone for advanced prostate cancer. $N$ Engl J Med. 2004;351(15):1502-1512. doi:10.1056/NEJMo a040720

25. Michels J, Montemurro T, Murray N, et al. First- and second-line chemotherapy with docetaxel or mitoxantrone in patients with hormone-refractory prostate cancer: does sequence matter? Cancer. 2006;106(5):1041-1046. doi:10.1002/(ISSN) 1097-0142

26. Kantoff PW, Higano CS, Shore ND, et al. Sipuleucel-T immunotherapy for castration-resistant prostate cancer. $N$ Engl J Med. 2010;363 (5):411-422. doi:10.1056/NEJMoa1001294

27. Oliver RTD, Williams G, Paris AMI, et al. Intermittent androgen deprivation after PSA-complete response as a strategy to reduce induction of hormone-resistant prostate cancer. Urology. 1997;49 (1):79-82. doi:10.1016/S0090-4295(96)00373-1 
28. Nishiyama T, Terunuma M. Hormonal sensitivity following endocrine withdrawal in hormone-refractory prostate cancer. Urol Int 2000;65(1):28-31. doi:10.1159/000064830

29. Sartor AO, Tangen CM, Hussain MHA, et al. Antiandrogen withdrawal in castrate-refractory prostate cancer: a Southwest Oncology Group trial (SWOG 9426). Cancer. 2008;112(11):2393-2400. doi:10.1002/(ISSN)1097-0142

30. Qu Y, Dai B, Kong Y, et al. Clinical analysis of docetaxel combined with prednisone in the treatment of metastatic castration-resistant prostate cancer. Chin J Urol. 2013;34(7):505-509.

31. Kellokumpu-Lehtinen PL, Harmenberg U, Joensuu T, et al. 2-weekly versus 3-weekly docetaxel to treat castration-resistant advanced prostate cancer: a randomised, phase 3 trial. Lancet Oncol. 2013;14 (2):117-124. doi:10.1016/S1470-2045(12)70537-5

32. Berthold DR, Pond GR, Soban F, et al. Docetaxel plus prednisone or mitoxantrone plus prednisone for advanced prostate cancer: updated survival in the TAX 327 study. J Clin Oncol. 2008;26(2):242-245. doi:10.1200/JCO.2007.12.4008

33. Finlay IG, Mason MD, Shelley M. Radioisotopes for the palliation of metastatic bone cancer: a systematic review. Lancet Oncol. 2005;6 (6):392-400. doi:10.1016/S1470-2045(05)70206-0

34. Pandit-Taskar N, Batraki M, Divgi CR. Radiopharmaceutical therapy for palliation of bone pain from osseous metastases. $\mathrm{J} \mathrm{Nucl} \mathrm{Med}$. 2004;45(8):1358-1365.

35. Parker C, Nilsson S, Heinrich D, et al. Alpha emitter radium-223 and survival in metastatic prostate cancer. $N$ Engl J Med. 2013;369 (3):213-223. doi:10.1056/NEJMoa1213755

36. Hoskin P, Sartor O, O'Sullivan JM, et al. Efficacy and safety of radium-223 dichloride in patients with castration-resistant prostate cancer and symptomatic bone metastases, with or without previous docetaxel use: a prespecified subgroup analysis from the randomised, double-blind, phase 3 ALSYMPCA trial. Lancet Oncol. 2014;15 (12):1397-1406. doi:10.1016/S1470-2045(14)70474-7

37. Parker C, Coleman RE, Nilsson S, et al. Updated survival, quality of life (QOL), and safety data of radium-223 chloride (RA-223) in patients with castration-resistant prostate cancer (CRPC) with bone metastases from the phase 3 double-blind, randomized, multinational study (ALSYMPCA). Ann Oncol. 2012;23:ix294. doi:10.1016/ S0923-7534(20)33503-1

38. Sternberg CN, Castellano D, Daugaard G, et al. Abiraterone acetate for patients with metastatic castration-resistant prostate cancer progressing after chemotherapy: final analysis of a multicentre, open-label, early-access protocol trial. Lancet Oncol. 2014;15 (11):1263-1268. doi:10.1016/S1470-2045(14)70417-6

39. Scher HI, Fizazi K, Saad F, et al. Increased survival with enzalutamide in prostate cancer after chemotherapy. $N$ Engl J Med. 2012;367 (13):1187-1197. doi:10.1056/NEJMoa1207506

40. de Bono JS, Oudard S, Ozguroglu M, et al. Prednisone plus cabazitaxel or mitoxantrone for metastatic castration-resistant prostate cancer progressing after docetaxel treatment: a randomized open-label trial. Lancet. 2010;376(9747):1147-1154. doi:10.1016/S01406736(10)61389-X

41. Sartor AO, Oudard S, Sengelov L, et al. Cabazitaxel vs docetaxel in chemotherapy-naive $(\mathrm{CN})$ patients with metastatic castration-resistant prostate cancer (mCRPC): a three-arm phase III study (FIRSTANA). $J$ Clin Oncol. 2016;34:Abstr 5006. doi:10.1200/JCO.2016.34. 15_suppl.5006

42. de Bono JS, Hardy-Bessard AC, Kim CS, et al. Phase III non-inferiority study of cabazitaxel (C) $20 \mathrm{mg} / \mathrm{m}^{2}$ (C20) versus $25 \mathrm{mg} / \mathrm{m}^{2}$ (C25) in patients (pts) with metastatic castration-resistant prostate cancer (mCRPC) previously treated with docetaxel (D) $J$ Clin Oncol. 2016;34:Abstr 5008. doi:10.1200/JCO.2016. 34.15_suppl.5008

43. Beltran H, Tagawa ST, Park K, et al. Challenges in recognizing treatment-related neuroendocrine prostate cancer. J Clin Oncol. 2012;30(36):e386-e389. doi:10.1200/JCO.2011.41.5166
44. Aggarwal R, Huang J, Alumkal JJ, et al. Clinical and genomic characterization of treatment-emergent small-cell neuroendocrine prostate cancer: a multi-institutional prospective study. J Clin Oncol. 2018;36(24):2492-2503. doi:10.1200/JCO.2017.77.6880

45. Fléchon A, Pouessel D, Ferlay C, et al. Phase II study of carboplatin and etoposide in patients with anaplastic progressive metastatic castration-resistant prostate cancer (mCRPC) with or without neuroendocrine differentiation: results of the French Genito-Urinary Tumor Group (GETUG) P01 trial. Ann Oncol. 2011;22 (11):2476-2481. doi:10.1093/annonc/mdr004

46. Gillessen S, Omlin A, Attard GA, et al. Management of patients with advanced prostate cancer: recommendations of the St Gallen Advanced Prostate Cancer Consensus Conference (APCCC) 2015. Ann Oncol. 2015;26(8):2354-2355. doi:10.1093/annonc/ $\operatorname{mdv} 360$

47. Scher HI, Morris MJ, Stadler WM, et al. Trial design and objectives for castration-resistant prostate cancer: updated recommendations from the Prostate Cancer Clinical Trials Working Group 3. J Clin Oncol. 2016;34 (12):1402-1418. doi:10.1200/JCO.2015.64.2702

48. Saad F, Gleason DM, Murray R, et al. Long-term efficacy of zoledronic acid for the prevention of skeletal complications in patients with metastatic hormone-refractory prostate cancer. J Natl Cancer Inst. 2004;96(11):879-882. doi:10.1093/jnci/djh141

49. Saad F, Gleason DM, Murray R, et al. A randomized, placebo-controlled trial of zoledronic acid in patients with hormone-refractory metastatic prostate carcinoma. J Natl Cancer Inst. 2002;94(19):1458-1468. doi:10.1093/jnci/94.19.1458

50. Fizazi K, Carducci M, Smith M, et al. Denosumab versus zoledronic acid for treatment of bone metastases in men with castration-resistant prostate cancer: a randomised, double-blind study. Lancet. 2011;377(9768):813-822. doi:10.1016/S01406736(10)62344-6

51. Porter AT, McEwan AJ. Strontium-89 as an adjuvant to external beam radiation improves pain relief and delays disease progression in advanced prostate cancer: results of a randomized controlled trial. Semin Oncol. 1993;20(3 Suppl 2):38-43.

52. Laing AH, Ackery DM, Bayly RJ, et al. Strontium-89 chloride for pain palliation in prostatic skeletal malignancy. $\mathrm{Br} J$ Radiol. 1991;64(765):816-822. doi:10.1259/0007-1285-64-765-816

53. Lewington VJ, McEwan AJ, Ackery DM, et al. A prospective, randomised double-blind crossover study to examine the efficacy of strontium-89 in pain palliation in patients with advanced prostate cancer metastatic to bone. Eur J Cancer. 1991;27:954. doi:10.1016/ 0277-5379(91)90257-E

54. Lee CK, Aeppli DM, Unger J, et al. Strontium-89 chloride (Metastron) for palliative treatment of bony metastases: the University of Minnesota experience. American Journal of Clinical Oncology. 1996;19 (2):102-107. doi:10.1097/00000421-199604000-00003

55. Serafini AN, Houston SJ, Resche I, et al. Palliation of pain associated with metastatic bone cancer using samarium-153 lexidronam: a double-blind placebo-controlled study. J Clin Oncol. 1998;16 (4):1574-1581. doi:10.1200/JCO.1998.16.4.1574

56. Sartor O, Reid RH, Hoskin PJ, et al. Samarium-153-lexidronam complex for the treatment of painful bone metastases in hormone refractory prostate cancer. Urology. 2004;63(5):940-945. doi:10.10 16/j.urology.2004.01.034

57. Sartor O, Reid RH, Bushnell DL, et al. Safety and efficacy of repeat administration of samarium sm-153 lexidronam to patients with metastatic bone pain. Cancer. 2007;109(3):637-643. doi:10.1002/ (ISSN)1097-0142

58. Oosterhof GO, Roberts JT, de Reijke TM, et al. Strontium (89) chloride versus palliative local field radiotherapy in patients with hormonal escaped prostate cancer: a phase III study of the European Organisation for Research and Treatment of Cancer, Genitourinary Group. Eur Urol. 2003;44(5):519-526. doi:10.1016/ S0302-2838(03)00364-6 
59. Singh D, Yi WS, Brasacchio RA, et al. Is there a favorable subset of patients with prostate cancer who develop oligometastases? Int J Radiat Oncol Biol Phys. 2004;58(1):3-10. doi:10.1016/S03603016(03)01442-1

60. Ost P, Bossi A, Decaestecker K, et al. Metastasis-directed therapy of regional and distant recurrences after curative treatment of prostate cancer: a systematic review of the literature. Eur Urol. 2015;67 (5):852-863. doi:10.1016/j.eururo.2014.09.004

61. Prostate Cancer Working Group of China Anti-Cancer Association Genitourinary Cancer Committee. Expert consensus on genetic testing in Chinese prostate cancer patients (2018 edition). China Oncol. 2018;28(8):627-633.
62. Wei $\mathrm{Y}, \mathrm{Wu} J, \mathrm{Gu} \mathrm{W}$, et al. Germline DNA repair gene mutation landscape in Chinese prostate cancer patients. Eur Urol. 2019;76 (3):280-283. doi:10.1016/j.eururo.2019.06.004

63. Professional Committee of Urological and Male Reproductive System Tumor, Chinese Anti-Cancer Association, China Cancer Hospital, Urological Oncology Group. Chinese expert consensus on the organization and implementation of comprehensive diagnosis and treatment of urogenital tumors. China Oncol. 2017;27(11):917-992.

\section{Publish your work in this journal}

Cancer Management and Research is an international, peer-reviewed open access journal focusing on cancer research and the optimal use of preventative and integrated treatment interventions to achieve improved outcomes, enhanced survival and quality of life for the cancer patient.
The manuscript management system is completely online and includes a very quick and fair peer-review system, which is all easy to use. Visit http://www.dovepress.com/testimonials.php to read real quotes from published authors. 